International Journal of Pure and Applied Mathematics

Volume 112 No. 3 2017, 557-569

ISSN: 1311-8080 (printed version); ISSN: 1314-3395 (on-line version)

url: http://www.ijpam.eu

doi: 10.12732 /ijpam.v112i3.8

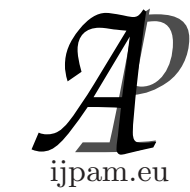

\title{
NUMERICAL SOLUTIONS OF THE FORCED PERTURBED KORTEWEG-DE VRIES EQUATION WITH VARIABLE COEFFICIENTS
}

\author{
Kim Gaik Tay ${ }^{1}$, Yaan Yee Choy $^{2}$, Wei King Tiong ${ }^{3}$ \\ Chee Tiong Ong ${ }^{4}$, Nazatulsyima Mohd Yazid ${ }^{5}$ \\ ${ }^{1}$ Department of Communication Engineering \\ University of Tun Hussein Onn Malaysia \\ 86400, Batu Pahat, Johor, MALAYSIA \\ ${ }^{2}$ Department of Mathematics and Statistics \\ University of Tun Hussein Onn Malaysia \\ 86400, Batu Pahat, Johor, MALAYSIA \\ ${ }^{3}$ Department of Computational Science and Mathematics \\ University of Malaysia Sarawak \\ Jalan Dato Mohd Musa, 94300 Kota Samarahan, Sarawak, MALAYSIA \\ ${ }^{4}$ Department of Mathematics \\ University of Technology Malaysia \\ 81310 Skudai, Johor, MALAYSIA \\ ${ }^{5}$ Department of Mathematics and Statistics \\ University of Tun Hussein Onn Malaysia \\ 86400, Batu Pahat, Johor, MALAYSIA
}

\begin{abstract}
In this paper, we solved the forced perturbed Korteweg-de vries (FpKdV) with variable coefficient arises in nonlinear wave propagation in an elastic tube filled with a symmetrical stenosis filled with a viscous fluid by two numerical methods, namely method of lines and pseudospectral method. We then compared both numerical solution with its progressive wave solution. Both methods solve FpKdV equation with maximum absolute errors of $10^{-2}$.
\end{abstract}

$\begin{array}{lr}\text { Received: } & \text { October 23, } 2016 \\ \text { Revised: } & \text { December 18, } 2016 \\ \text { Published: } & \text { February 9, } 2017\end{array}$

(C) 2017 Academic Publications, Ltd. url: www.acadpubl.eu 


\section{AMS Subject Classification: $65 \mathrm{~N} 40$}

Key Words: Korteweg-de Vries equation, perturbed Korteweg-de Vries equation, Forced perturbed Korteweg-de Vries equation, method of lines, pseudospectral method

\section{Introduction}

Tay [1] studied small-but-finite amplitude weakly nonlinear wave propagation in a prestressed fluid-filled elastic tube with a symmetrical setenosis filled with a Newtonian fluid by applying the reductive perturbation method in the long wave approximation. By using the stretched coordinate of boundary-value type and extending the field quantities into the asymptotic series of order $\epsilon$, where $\epsilon$ is a small parameter, the governing equations are reduced to the Forced perturbed Korteweg-de Vries (FpKdV) equation with variable coefficients

$$
U_{\tau}+\mu_{1} U U_{\xi}-\mu_{3} U_{\xi \xi \xi}-\mu_{4}(\tau) U_{\xi}+\mu_{5} U=\mu(\tau),
$$

where $U$ is the radial displacement, $\xi$ is a temporal variable, $\tau$ is a spatial variable, and $\mu_{1}, \mu_{3}, \mu_{4}(\tau), \mu_{5}$ and $\mu(\tau)$ are the coefficients of nonlinearity, dispersion, variable coefficient, perturbed and forcing term respectively. The presence of forcing term, $\mu(\tau)$ and variable coefficient term, $\mu_{4}(\tau) U_{\xi}$ are due to the presence of stenosis, while the perturbed term $\mu_{5} U$ is caused by Newtonian fluid. The coefficients of $\mu_{1}, \mu_{3}, \mu_{4}(\tau), \mu_{5}$ and $\mu(\tau)$ are defined by [1] as

$$
\begin{gathered}
\mu_{1}=\frac{5}{2 \lambda_{\theta}}+\frac{\beta_{2}}{\beta_{1}}, \quad \mu_{3}=\frac{\beta_{0}}{2 \beta_{1}}-\frac{m}{4 \lambda_{z}}, \\
\mu_{4}(\tau)=-\frac{\lambda_{\theta} \gamma_{2}}{\beta_{1}} G(\tau)+\left[\frac{\beta_{2}}{\beta_{1}}+\frac{1}{2 \lambda_{\theta}}\right] g(\tau), \\
\mu_{5}=\frac{4 \nu}{c \lambda_{\theta}^{2}}, \quad \mu(\tau)=\frac{1}{2} g^{\prime}(\tau)-\frac{\lambda_{\theta} \gamma_{1}}{2 \beta_{1}} G^{\prime}(\tau),
\end{gathered}
$$

where

$$
\begin{aligned}
& \gamma_{0}=\frac{1}{\lambda_{\theta} \lambda_{z}}\left(\lambda_{\theta}-\frac{1}{\lambda_{\theta}^{3} \lambda_{z}^{2}}\right) F\left(\lambda_{\theta}, \lambda_{z}\right), \\
& \gamma_{1}=\frac{1}{\lambda_{\theta} \lambda_{z}}\left[1+\frac{3}{\lambda_{\theta}^{4} \lambda_{z}^{2}}+2 \alpha\left(\lambda_{\theta}-\frac{1}{\lambda_{\theta}^{3} \lambda_{z}^{2}}\right)^{2}\right] F\left(\lambda_{\theta}, \lambda_{z}\right), \\
& \gamma_{2}=\frac{1}{2 \lambda_{\theta} \lambda_{z}}\left[-\frac{12}{\lambda_{\theta}^{5} \lambda_{z}^{2}}+6 \alpha\left(\lambda_{\theta}-\frac{1}{\lambda_{\theta}^{3} \lambda_{z}^{2}}\right)\left(1+\frac{3}{\lambda_{\theta}^{4} \lambda_{z}^{2}}\right)\right.
\end{aligned}
$$




$$
\begin{gathered}
\left.+4 \alpha^{2}\left(\lambda_{\theta}-\frac{1}{\lambda_{\theta}^{3} \lambda_{z}^{2}}\right)^{3}\right] F\left(\lambda_{\theta}, \lambda_{z}\right), \\
\beta_{0}=\frac{1}{\lambda_{\theta}}\left(\lambda_{z}-\frac{1}{\lambda_{\theta}^{2} \lambda_{z}^{3}}\right) F\left(\lambda_{\theta}, \lambda_{z}\right), \quad \beta_{1}=\gamma_{1}-\frac{\gamma_{0}}{\lambda_{\theta}}, \quad \beta_{2}=\gamma_{2}-\frac{\beta_{1}}{\lambda_{\theta}},
\end{gathered}
$$

given that $F\left(\lambda_{\theta}, \lambda_{z}\right)=\exp \left[\alpha\left(\lambda_{\theta}^{2}+\lambda_{z}^{2}+\frac{1}{\lambda_{\theta}^{2} \lambda_{z}^{2}}-3\right)\right], \alpha=1.948, \lambda_{\theta}=\lambda_{z}=$ 1.6, $\nu=1, c=15.391, m=0.1, G(\tau)=0$ and $g(\tau)=\operatorname{sech}(0.01 \tau)$. Here $\alpha$ refers to material constant, $\lambda_{\theta}$ is the initial circumferential stretch ratio, $\lambda_{z}$ is the initial axial stretch ratio, $\nu$ is kinematic viscosity, $m$ is mass of artery and $c$ is the scale parameter.

Notice that when the forcing term becomes zero, equation (1) reduces to the perturbed Korteweg-de Vries equation arises in nonlinear wave propagation in a prestressed thin elastic tube filled with viscous fluid [2] or layered fluid [3].

$$
U_{\tau}+\mu_{1} U U_{\xi}-\mu_{3} U_{\xi \xi \xi}-\mu_{4}(\tau) U_{\xi}+\mu_{5} U=0
$$

When $\mu_{5}=0$ and $\mu_{4}(\tau)=0$, equation (4) reduces to the standard Korteweg-de Vries $(\mathrm{KdV})$ equation,

$$
U_{\tau}+\mu_{1} U U_{\xi}+\mu_{3} U_{\xi \xi \xi}
$$

The KdV equation (5) was first introduced by Korteweg-and Vries [4] to describe the evolution of long, one-dimensional shallow water waves with small but finite amplitude. In 1965, Zabusky and Kruskal [5] discovered the concept of the solitons while studying the results of a numerical computation on the KdV equation. Since then, the KdV equation has been found to describe many physical phenomena, including long internal waves in ocean, magneto hydrodynamics waves in warm plasma, ion acoustic waves in a plasma, acoustic waves on a crystal lattice and wave propagation in an elastic tube filled with an inviscid fluid.

Many studies have been devoted to the numerical solutions of the KdV equation (5), however, none of the literature works dealt with numerical solutions of the FpKdV equation (1). Motivated with the works of wave propagation which yielded the FpKdV equation (1) as well as numerical methods, we are going to find numerical solution of the FpKdV by two numerical methods, namely method of line (MOL) and pseudospectral. The numerical solution of the Fp$\mathrm{KdV}$ equation is then compared in terms of its maximum absolute error at certain space with progressive wave solution conducted by [1]. 


\section{The MOL}

The MOL is a powerful method used to solve partial differential equations (PDEs). It replaces the spatial derivatives by finite-difference approximation and reducing the PDEs into time dependent system of ODEs [6]-[7]. This system of ODEs can be solved by using time integrator such as fourth-order Runge-Kutta (RK4) method.

MOL has been widely used to solve the nonlinear evolution equations such as Korteweg-de Vries (KdV) equation [8], extended nonlinear KdV equation, good Boussinesq equation, fifth-order KaupKupershmidt equation and an extended fifth-order Korteweg-de Vries (KdV5) equation [9], delay differential equations [10], two-dimensional sine-Gordon equation [11], the Nwogu one-dimensional extended Boussinesq equation [12].

In this paper, the temporal derivatives $\xi$ in (1) are firstly discretized using central finite difference formulae as follows:

$$
U_{\xi} \approx \frac{U_{i+1}-U_{i-1}}{2 \Delta \xi}, \quad U_{\xi \xi \xi} \approx \frac{U_{i+2}-2 U_{i+1}+2 U_{i-1}-U_{i-2}}{2(\Delta \xi)^{3}},
$$

$i$ is the index denoting the temporal position along $\xi$-axis and $\Delta \xi$ is the step size along the axis. The $\xi$-interval is divided into $M$ points with $i=1,2, \ldots, M-$ $1, M$. Therefore, the MOL approximation of (1) is given by

$$
\begin{aligned}
\frac{\partial U_{i}}{\partial \tau}= & -\frac{\mu_{1}}{2 \Delta \xi} U_{i}\left(U_{i+1}-U_{i-1}\right)+\frac{\mu_{3}}{2(\Delta \xi)^{3}}\left(U_{i+2}-2 U_{i+1}+2 U_{i-1}-U_{i-2}\right) \\
& +\frac{\mu_{4}(\tau)}{2 \Delta \xi}\left(U_{i+1}-U_{i-1}\right)-\mu_{5} U_{i}+\mu(\tau) \equiv f\left(U_{i}\right) .
\end{aligned}
$$

Since there is only one independent variable, which is $\tau$, hence equation (7) is an ODE. Besides, since $i$ varies from 1, 2, up to $M$, thus equation (7) represents a system of $M$ equations of ODEs with the initial condition given by

$$
U\left(\xi_{i}, \tau=0\right)=U_{0}\left(\xi_{i}\right), \quad i=1,2, \ldots, M-1, M
$$

For the space integration, the RK4 method is applied. Thus, the numerical solution at space $\tau_{i+1}$ is

$$
U_{i}^{j+1}=U_{i}^{j}+\frac{1}{6}\left(a_{i}^{j}+2 b_{i}^{j}+2 c_{i}^{j}+d_{i}^{j}\right),
$$


where

$$
\begin{aligned}
a_{i}^{j} & =\Delta \tau f\left(U_{i}^{j}\right), \\
b_{i}^{j} & =\Delta \tau f\left(U_{i}^{j}+\frac{1}{2} a_{i}^{j}\right), \\
c_{i}^{j} & =\Delta \tau f\left(U_{i}^{j}+\frac{1}{2} b_{i}^{j}\right), \\
d_{i}^{j} & =\Delta \tau f\left(U_{i}^{j}+c_{i}^{j}\right) .
\end{aligned}
$$

Here $\Delta \tau$ is the step size of the spatial coordinate.

\section{The Pseudospectral Method}

Pseudospectral method transforms the spatial derivatives of the PDEs by Fourier transform and substitutes the temporal derivative by finite-difference approximation which yields a 3-level scheme to be solved numerically. It has been used to solve the $\mathrm{KdV}$ [13], Burgers [14] and KdV-Burgers equations [15].

The Chan and Kerkhoven [13] scheme is extended for the FpKdV equation (1). The FpKdV equation (1) is integrated in space $\tau$ by the leapfrog finitedifference scheme in the spectral time $\xi$. The infinite interval is replaced by $-L<\xi<L$ with $L$ sufficiently large such that the periodicity assumptions hold.

By introducing $\zeta=s \xi+\pi$, where $s=\pi / L, U(\xi, \tau)$ will be transformed into $V(\zeta, \tau)$ as

$$
\frac{\partial V}{\partial \tau}+\mu_{1} s V \frac{\partial V}{\partial \zeta}-\mu_{3} s^{3} \frac{\partial^{3} V}{\partial \zeta^{3}}-\mu_{4}(\tau) s \frac{\partial V}{\partial \zeta}+\mu_{5} V=\mu(\tau)
$$

By letting $W(\zeta, \tau)=\frac{1}{2} s V^{2}$, the nonlinear term in equation (11) reduces to

$$
\frac{\partial V}{\partial \tau}+\mu_{1} \frac{\partial W}{\partial \zeta}-\mu_{3} s^{3} \frac{\partial^{3} V}{\partial \zeta^{3}}-\mu_{4}(\tau) s \frac{\partial V}{\partial \zeta}+\mu_{5} V=\mu(\tau) .
$$

In order to obtain the numerical solution of (12), the interval $[0,2 \pi]$ is discretised by $N+1$ equidistant points. Let $\zeta_{0}=0, \zeta_{1}, \zeta_{2}, \ldots, \zeta_{N}=2 \pi$, so that $\Delta \zeta=\frac{2 \pi}{N}$. In this case, $N$ is power of two, let $m=\frac{N}{2}$, the Discrete Fourier Transform (DFT) of $V\left(\zeta_{j}, \tau\right)$ for $j=0,1,2, \ldots, N-1$, denoted by $\hat{V}(p, \tau)$ is given by

$$
\hat{V}(p, \tau)=\frac{1}{\sqrt{N}} \sum_{j=0}^{N-1} V\left(\zeta_{j}, \tau\right) e^{-\left(\frac{2 \pi j p}{N}\right) i}
$$


where $p=-m,-m+1,-m+2, \ldots, m-1$ and $i=\sqrt{-1}$, the usual imaginary number. The inverse Fourier transform of $\hat{V}(p, \tau)$ for $p=-m,-m+1,-m+$ $2, \ldots, m-1$, denoted by $V\left(\xi_{j}, \tau\right)$ can be written as

$$
V\left(\xi_{j}, \tau\right)=\frac{1}{\sqrt{N}} \sum_{p=-m}^{m-1} \hat{V}(p, \tau) e^{\left(\frac{2 \pi j p}{N}\right) i}
$$

where $j=0,1,2, \ldots, N-1$.

Then, DFT of (12) with respect to $\zeta$ gives

$$
\begin{aligned}
& \frac{\partial \hat{V}(p, \tau)}{\partial \tau}+i \mu_{1} p \hat{W}(p, \tau)+i \mu_{3}(s p)^{3} \hat{V}(p, \tau) \\
& -i \mu_{4}(\tau) \operatorname{sp} \hat{V}(p, \tau)+\mu_{5} \hat{V}(p, \tau)=\mu(\tau),
\end{aligned}
$$

where the cap stands for the Fourier transform.

By using the following approximations

$$
\begin{aligned}
& \frac{\partial \hat{V}(p, \tau)}{\partial \tau} \approx \frac{\hat{V}(p, \tau+\Delta \tau)-\hat{V}(p, \tau-\Delta \tau)}{2 \Delta \tau}=\frac{\hat{V}^{k+1}-\hat{V}^{k-1}}{2 \Delta \tau}, \\
& \hat{V}(p, \tau) \approx \frac{\hat{V}(p, \tau+\Delta \tau)+\hat{V}(p, \tau-\Delta \tau)}{2}=\frac{\hat{V}^{k+1}+\hat{V}^{k-1}}{2} .
\end{aligned}
$$

On equation (13), this reduces to the forward scheme given by

$$
\begin{aligned}
& \quad \hat{V}^{k+1}= \\
& \frac{\hat{V}^{k-1}\left[1-i \Delta \tau \mu_{3} s^{3} p^{3}+i \Delta \tau \mu_{4}(\tau) s p-\Delta \tau \mu_{5}\right]-2 i \Delta \tau \mu_{1} p \hat{W}(p, \tau)+2 \Delta \tau \mu(\tau)}{\left[1+i \Delta \tau \mu_{3} s^{3} p^{3}-i \Delta \tau \mu_{4}(\tau) s p+\Delta \tau \mu_{5}\right]} .
\end{aligned}
$$

Equation (15) is a three-level scheme, in which one needs to know the first level, initial condition that is $\hat{V}^{k-1}$ and subsequent second level, $\hat{V}^{k}$, then one can get the third level, $\hat{V}^{k+1}$. The process is repeated till the desired $\hat{V}^{k+1}$ is obtained. To get the second level, $\hat{V}^{k}$, the interval between $\hat{V}^{k-1}$ and $\hat{V}^{k}$ is divided by ten sub intervals. Later, we substitute $\Delta \tau$ in (15) by $\Delta \tau / 10$ in order to get the equation for $\hat{V}^{k}$ as

$$
\begin{aligned}
& \hat{V}^{k}= \\
& \frac{\hat{V}^{k-1}\left[1-i \frac{\Delta \tau}{10} \mu_{3} s^{3} p^{3}+i \frac{\Delta \tau}{10} \mu_{4}(\tau) s p-\frac{\Delta \tau}{10} \mu_{5}\right]-2 i \frac{\Delta \tau}{10} \mu_{1} p \hat{W}(p, \tau)+2 \frac{\Delta \tau}{10} \mu(\tau)}{\left[1+i \frac{\Delta \tau}{10} \mu_{3} s^{3} p^{3}-i \frac{\Delta \tau}{10} \mu_{4}(\tau) s p+\frac{\Delta \tau}{10} \mu_{5}\right]} .
\end{aligned}
$$


Equation (16) is evaluated for ten times to get $\hat{V}^{k}$, since the interval between $\hat{V}^{k-1}$ and $\hat{V}^{k}$, is divided by ten sub intervals.

\section{Progressive Wave Solution}

The progressive wave solution of the FpKdV equation as given by [1] is

$$
U=-a_{0} \exp \left(-\frac{4}{3} \mu_{5} \tau\right) \operatorname{sech}^{2} \zeta+\exp \left(-\mu_{5} \tau\right) \int_{0}^{\tau} \mu(s) \exp \left(\mu_{5} s\right) d s
$$

where $a_{0}$ is a constant. The phase function $\zeta$ can be expressed as

$$
\begin{array}{r}
\zeta=\left(\frac{\mu_{1} a}{12 \mu_{3}}\right)^{\frac{1}{2}}\left\{\xi+\frac{\mu_{1} a_{0}}{4 \mu_{5}}\left[1-\exp \left(-\frac{4}{3} \mu_{5} \tau\right)\right] \tau\right. \\
\left.-\int_{0}^{\tau}\left[-\mu_{4}(q)+\mu_{1} \exp \left(-\mu_{5} q\right) \int_{0}^{q} \mu(s) \exp \left(\mu_{5} s\right) d s\right] d q\right\} .
\end{array}
$$

\section{Results and Discussion}

For both numerical methods, we need the initial condition to start the numerical simulations. By letting $\tau=0$ in (17) and $a_{0}=1$, we use the initial condition as

$$
U=-\operatorname{sech}^{2} \sqrt{\frac{\mu_{1}}{12 \mu_{3}}} \xi .
$$

To calculate the accuracy of the numerical solution with the progressive wave solution, the maximum absolute errors between the progressive wave and numerical solutions are calculated based on the formula:

$$
L_{\infty}={ }_{\max }\left|U_{\text {progressive }}-U_{\text {numerical }}\right| \cdot
$$

Figure 1 (a) gives the MOL solution of the FpKdV equation (1) with time $\xi$ at certain space $\tau$, while Figure 1 (b) represents the progressive wave solution of the FpKdV equation (1) with time parameter $\xi$ at certain space $\tau$. MOL produces errors at the bottom of the wave (horizontal axis) if compared to the progressive wave solution. The solution of the FpKdV equation (1) with time $\xi$ shows the initial wave propagates to the left as space $\tau$ increases with decreasing amplitude. The overlapped graphs of both MOL and progressive wave solutions of the FpKdV equation are given in Figure 2. It is seen that 

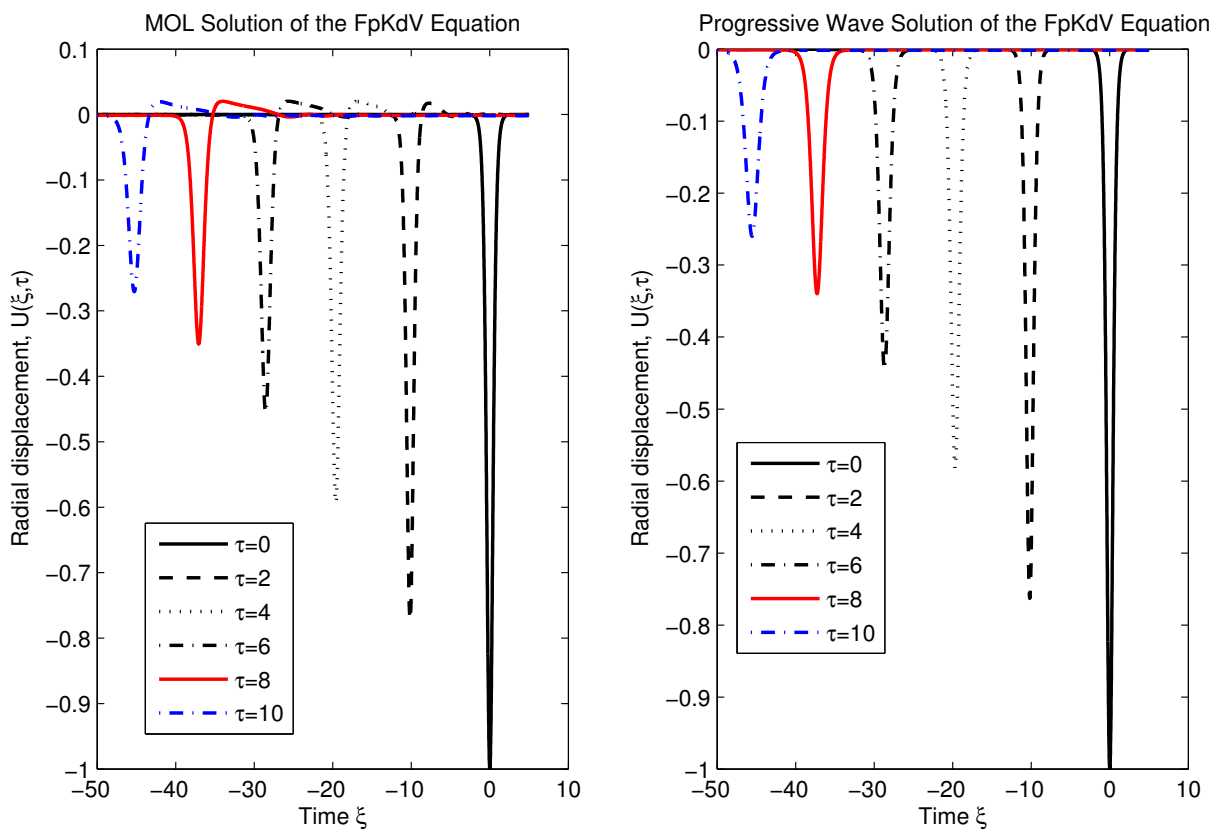

Figure 1: (a) MOL and (b) Progressive Wave Solutions of the FpKdV equation versus Time $\xi$ for different Space $\tau$ with $\Delta \xi=0.01, \Delta \tau=$ $1 x 10^{-6}$

MOL approximate progressive wave solution except at the bottom and is a bit run from its position.

The absolute error between the MOL and progressive wave solution of the FpKdV equation with variable coefficient at each space $\tau$ are given in Figure 3. The maximum absolute errors between the progressive wave and MOL solutions for each discretized temporal point at certain space $\tau$ are calculated based on the formula (20). Table-1 gives the maximum absolute error between the progressive wave solution and MOL solution for each discretized temporal point at certain space $\tau$. It shows the maximum absolute errors are in order of $10^{-2}$.

Figure 4(a) gives the pseudospectral solution of the FpKdV equation (1) with time $\xi$ at certain space $\tau$, while Figure 4(b) shows the progressive wave solution of the FpKdV equation (1) with time parameter $\xi$ at certain space $\tau$. Again, the pseudospectral solution of the FpKdV equation (1) with time $\xi$ produces error at the bottom of the wave if compared to the progressive wave 


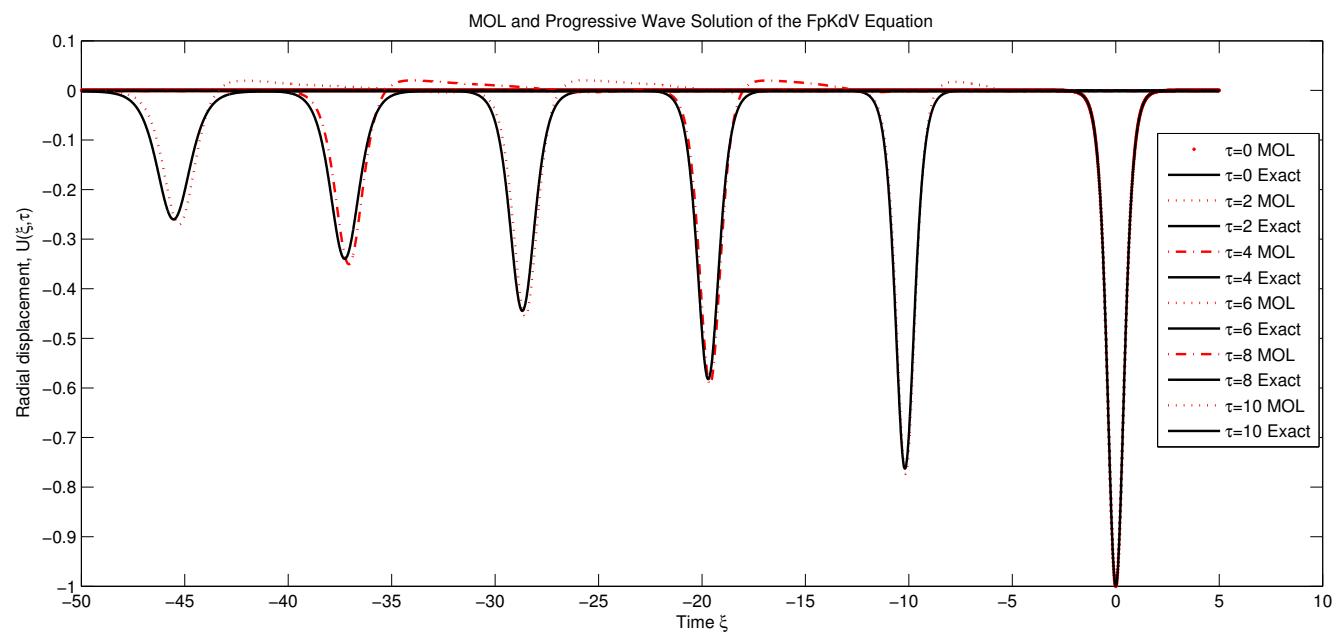

Figure 2: MOL and Progressive Wave Solutions of the FpKdV equation versus Time $\xi$ for different Space $\tau$ with $\Delta \xi=0.01, \Delta \tau=1 \times 10^{-6}$

Table 1: Maximum absolute error of the FpKdV equation for different space $\tau$ by MOL method

\begin{tabular}{|c|c|c|c|c|c|c|}
\hline Space, $\tau$ & $\mathbf{0}$ & $\mathbf{2}$ & $\mathbf{4}$ & $\mathbf{6}$ & $\mathbf{8}$ & $\mathbf{1 0}$ \\
\hline$L_{\infty}$ & 0 & 0.0387 & 0.0576 & 0.0636 & 0.0620 & 0.0563 \\
\hline
\end{tabular}

solution. Both pseudospectral and progressive wave solution of the FpKdV equation are overlapped as given in Figure 5. It shows that pseudospectral method approximates the FpKdV equation solution except at the bottom and is a bit run from its position when $\tau=8$ and $\tau=10$.

The graph of the absolute error between the progressive wave and pseudospectral solutions of the FpKdV equation for each discretized time $\xi$ point at certain space $\tau$ is shown in Figure 6 . The maximum absolute error between the progressive wave and pseudospectral solutions of the FpKdV equation for each discretized time $\xi$ point at certain space $\tau$ are calculated based on the formula (20) and shown in Table 2. It shows that the maximum absolute errors are in order of $10^{-2}$. 

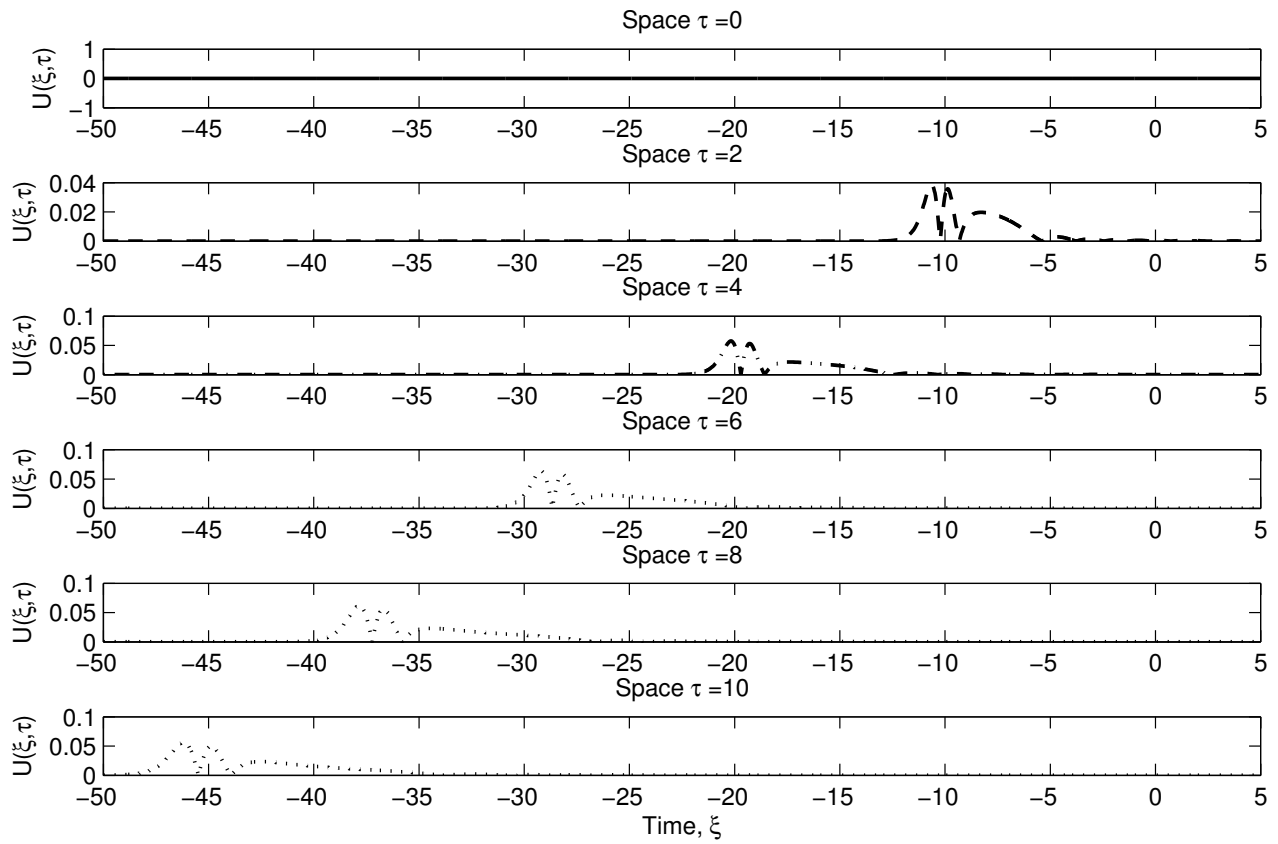

Figure 3: Absolute error between MOL and Progressive Wave Solutions of the FpKdV equation versus Time $\xi$ for different Space $\tau$

Table 2: Maximum absolute error of the FpKdV equation for different space $\tau$ by pseudospectral method

\begin{tabular}{|c|c|c|c|c|c|c|}
\hline Space, $\tau$ & $\mathbf{0}$ & $\mathbf{2}$ & $\mathbf{4}$ & $\mathbf{6}$ & $\mathbf{8}$ & $\mathbf{1 0}$ \\
\hline$L_{\infty}$ & 0 & 0.0346 & 0.0560 & 0.0654 & 0.0661 & 0.0624 \\
\hline
\end{tabular}



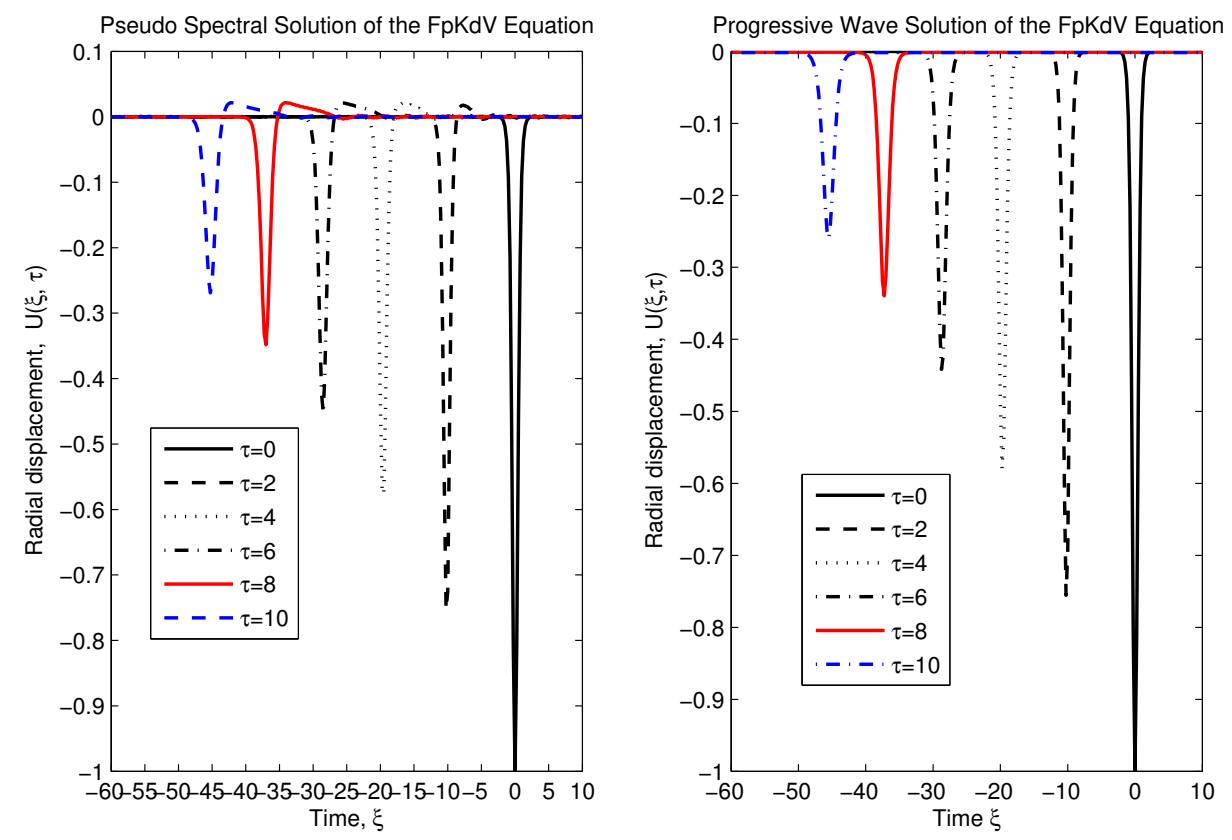

Figure 4: (a) Pseudospectral and (b) Progressive Wave Solutions of the FpKdV equation versus Time $\xi$ for different Space $\tau$ 


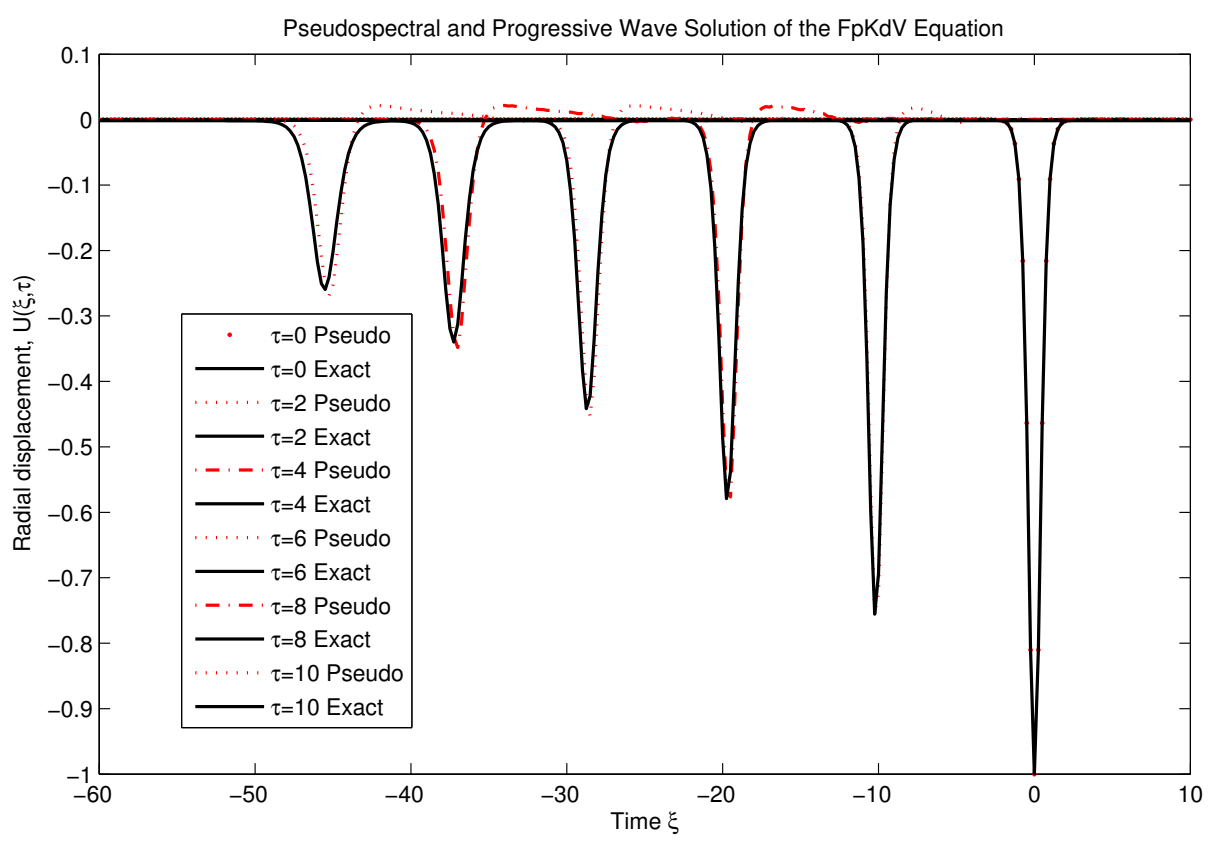

Figure 5: Pseudo spectral and Progressive Wave Solutions of the Fp$\mathrm{KdV}$ equation versus Time $\xi$ for different Space $\tau$

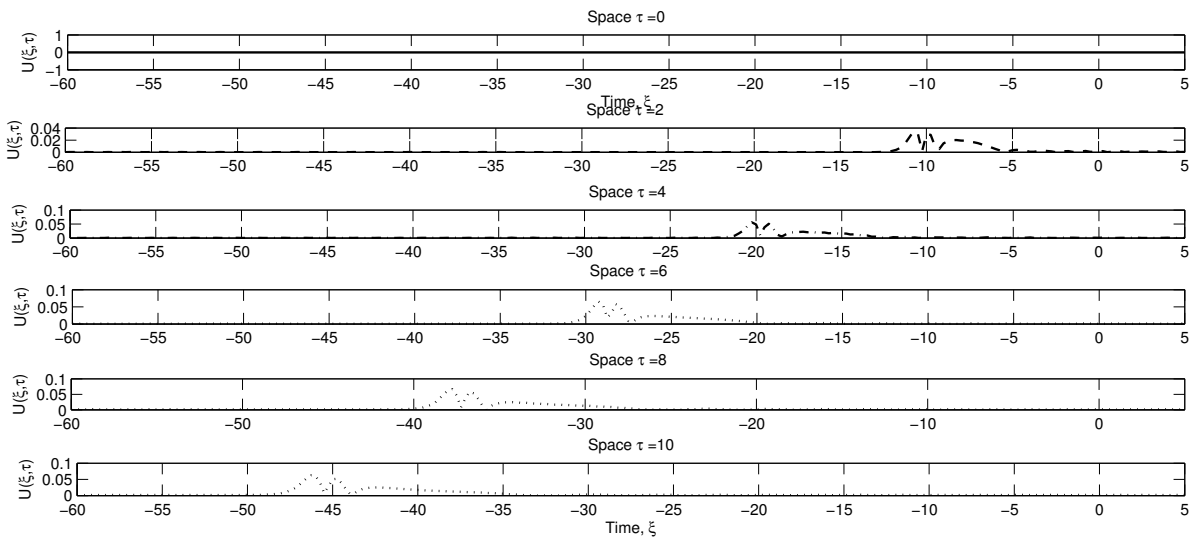

Figure 6: Absolute error between pseudospectral and Progressive Wave Solutions of the FpKdV equation versus Time $\xi$ for different Space $\tau$ 


\section{Conclusion}

We have solvde the FpKdV equation by using the MOL and pseudospectral method. The MOL and pseudospectral method solutions of the FpKdV equation (1) were plotted versus the progressive wave solution. From the observation, it was found that there were no differences for both MOL, pseudospectral and progressive wave solutions in terms of their amplitude and position. Both methods can solve the FpKdV equation with maximum absolute error in order of $10^{-2}$.

\section{References}

[1] K.G. Tay, C.T. Ong and N.M Mohd, Forced Perturbed Korteweg-de Vries Equation In An Elastic Tube Filled With A Viscous Fluid, International Journal of Engineering Science, 45, No.2-8 (2007), 339-349, doi: 10.1016/j.ijengsci.2007.03.017.

[2] H. Demiray, Slowly varying solitary waves in an elastic tube filled with a viscous fluid, ARI - An International Journal for Physical and Engineering Sciences, 51, No.1 (1998), 98-102, doi: 10.1007/s007770050039.

[3] H. Demiray, Solitary waves in elastic tubes filled with a layered fluid, International Journal of Engineering Science, 39, No.6 (2001), 629-639, doi: 10.1016/S0020-7225(00)000690 .

[4] D.J. Korteweg and G. de Vries, On the change of form long waves advancing in a rectangular canal and on a new type of long stationary waves, Philos. Magazine Series 5, 39, No.240 (1895), 422-443, doi: 10.1080/14786449508620739.

[5] N.J. Zabusky and M.D. Kruskal, Interaction of Solitons in a collisionless plasma and the recurrence of initial state, Physical Review Letters, 15, No.6 (1965), 240-243, doi: 10.1103/PhysRevLett.15.240.

[6] G. Hall and J.M. Watt, Modern Numerical Methods for Ordinary Differential Equations, Clarendon Press, Oxford (1976).

[7] W.Schiesser, The numerical method of lines: Integration of partial differential equations. Academic Press, San Diego (1991).

[8] W. Schiesser, Method of lines solution of the Korteweg-de Vries equation, Computers and Mathematics with Applications, 28, No.10-12 (1994), 147-154, doi: 10.1016/08981221(94)00190-1.

[9] P. Saucez, A. V Wouwer, W. E Schiesser and P. Zegeling, Method of lines study of nonlinear dispersive waves, Journal of Computational and Applied Mathematics, 168 , No.1-2 (2004), 413-423, doi: 10.1016/j.cam.2003.12.012.

[10] T. Koto, Method of lines approximations of delay differential equations, Computers and Mathematics with Applications, 48, No.1-2 (2004), 45-59, doi: 10.1016/j.camwa.2004.01.003.

[11] A.G, Bratsos, The solution of the two-dimensional sine-Gordon equation using the method of lines, Journal of Computational and Applied Mathematics, 206, No. 1 (2007), 251-277, doi: 10.1016/j.cam.2006.07.002. 
[12] S. Hamdi, W.H. Enright, Y. Ouellet, and W.E. Schiesser, Method of lines solutions of the extended Boussinesq equations, Journal of Computational and Applied Mathematics, 183, No.2 (2005), 327-342, doi: 10.1016/j.cam.2004.12.036.

[13] T.F. Chan and T. Kerhoven, Fourier methods with extented stability intervals for the Korteweg-de Vries equation, SIAM Journal of Numerical Analysis, 22, No.3 (1985), 441454.

[14] C.T. Ong, Y.M. Chew and K.G. Tay, Numerical Solution of the Burgers Equation, Book Chapter-10 of Advances in Theoretical and Numerical Methods, Penerbit UTM, Skudai (2007).

[15] Y.M. Chew, A Simulation Study of The Korteweg-de Vries-Burgers Equation, Universiti Teknologi Malaysia, M. Sci. Thesis (2004). 